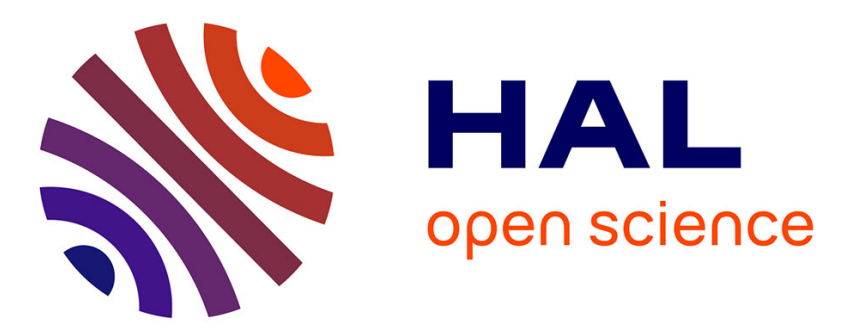

\title{
A low cost approach to acoustic filters acting as GPR cooperative targets for passive sensing
}

\author{
Jean-Michel Friedt, Arthur Hugeat
}

\section{To cite this version:}

Jean-Michel Friedt, Arthur Hugeat. A low cost approach to acoustic filters acting as GPR cooperative targets for passive sensing. 8 th International Workshop on Advanced Ground Penetrating Radar IWAGPR20178, Jul 2015, Florence, Italy. hal-03198169

\section{HAL Id: hal-03198169 \\ https://hal.science/hal-03198169}

Submitted on 14 Apr 2021

HAL is a multi-disciplinary open access archive for the deposit and dissemination of scientific research documents, whether they are published or not. The documents may come from teaching and research institutions in France or abroad, or from public or private research centers.
L'archive ouverte pluridisciplinaire HAL, est destinée au dépôt et à la diffusion de documents scientifiques de niveau recherche, publiés ou non, émanant des établissements d'enseignement et de recherche français ou étrangers, des laboratoires publics ou privés. 


\title{
A low cost approach to acoustic filters acting as GPR cooperative targets for passive sensing
}

\author{
J.-M Friedt, A. Hugeat \\ FEMTO-ST Time \& Frequency, 26 rue de l'Épitaphe, Besançon, France \\ Email: jmfriedt@femto-st.fr
}

\begin{abstract}
Ground Penetrating RADAR (GPR) is a classical sub-surface analysis tool widely used in civil engineering and environmental investigations. Currently limited to monitoring passive buried dielectric and conductivity interfaces, the instrument is perfectly suited to probe cooperative targets made of piezoelectric substrates designed to convert the incoming electromagnetic wave to an acoustic wave. The surface acoustic wave (SAW) velocity is strongly dependent on the physical environment of the transducer, allowing for sensing such quantities as temperature, stress or the presence of chemical compounds. In order to demonstrate the compatibility of SAW sensors acting as cooperative targets probed by GPR, we investigate the use of widely available commercial SAW filters for sensing purposes. Dedicated software for recording the Malå ProEx GPR signal and digital signal processing algorithms for extracting the physical quantity are demonstrated.
\end{abstract}

\section{INTRODUCTION}

Ground Penetrating RADAR (GPR) is classically used for detecting subsurface permittivity and conductivity interfaces. Being a general purpose pulse generator recording echoes, such an instrument is well suited for probing cooperative targets consisting of a target designed to return an echo whose delay is not only related to the distance to the emitter but also to a physical quantity. Initial investigations have consisted in using dielectric delay lines as sensing element, yielding large dimensions and little sensitivity [1], [2]. Converting the electromagnetic wave - propagating at a few hundreds of meters per microsecond - to an acoustic wave propagating at a few thousand meters per second, or a $10^{5}$ ratio - allows for shrinking the sensing element dimensions. More important, using single crystal piezoelectric substrates for this electromagnetic to mechanical conversion allows for a precise tuning of the dependence of the wave velocity with a given physical quantity (temperature [3], [4], strain [5], mass loading changing waveguide boundary condition for chemical sensing [6]). The devices converting an incoming electromagnetic signal to an acoustic (mechanical) wave and back to an electromagnetic signal after filtering is called a surface acoustic wave (SAW) filter. We have previously reported that dedicated SAW transducers are ideally suited as passive cooperative targets for sensing quantities through a wireless link when probed by GPR [7], [8]. However, cleanroom manufacturing of such devices requires dedicated design processes for producing the sensor, limiting the targeted audience to microelectronics development centers. In order to widen the interested audience to such technology, we demonstrate the use of commercially available SAW filters as sensing elements interrogated by GPR. We emphasize that no hardware modification is needed other than signal postprocessing of the recorded signals for recovering a physical quantity, here demonstrated on temperature sensing.

\section{Surface ACOUSTIC WAVE FILTER SELECTION}

Surface acoustic wave filters are manufactured by a single lithography processing step in which a thin metallic layer is deposited on a single crystal piezoelectric substrate, patterned in order to define a combshaped interdigitated electrode [9], [10]. Mirrors are patterned on the acoustic path as electrodes inducing acoustic velocity variation (boundary condition change due to the free to metalized surface inducing impedance change and hence reflecting a fraction of the incoming energy). This simple manufacturing process yields cost efficient production of such radiofrequency processing components, seen from a user perspective as an electrical dipole. However, operating in the radiofrequency range means that electrode dimensions are in the micrometer range due to the low acoustic velocity (typically in the thousands of meters per second, or 10 micrometer wavelength when considering operating frequencies in the hundreds of megahertz) and hence cleanroom manufacturing techniques are mandatory.

Radiofrequency filters are widely available from a number of commercial sources, including Epcos/TDK, Murata or Frec'n'sys SAS. We consider such filters as components for becoming familiar with acoustic transducers acting as GPR cooperative targets. Although not 
optimally designed for such a task, the signal is well defined and will be processed for a temperature sensing application. Indeed, the classical substrate for manufacturing wideband filters - lithium niobate - exhibits strong coupling and always a large temperature dependence of the acoustic velocity with temperature. A cooperative target recovers incoming electromagnetic energy through an antenna and sends back electromagnetic pulses through the same means: due to the tens of centimeter to meter wavelength involved in GPR operating conditions, an usual implementation is to connect a dipole antenna to electrodes patterned on the piezoelectric substrate: the SAW sensor is thus used in a dipole configuration of a reflective delay line. Although SAW filters are designed as 4-pole components for use in a transmission configuration, the unloaded output port exhibits impedance mismatch with the interdigitated transducer and hence a fraction of the incoming energy is reflected back to the port connected to an antenna. Using the filter as a reflective delay line is thus possible, with a delay indicated by the manufacturer in the datasheet in the microsecond range, well within the measurement range of commercial GPRs.

\section{Surface ACOUSTIC WAVE FILTERS AS GROUND PENETRATING RADAR COOPERATIVE TARGET}

Subsurface targets observed using GPR are typically located, in temperate or urban environments, in the first 10 meters or typically in the first $200 \mathrm{~ns}$ after the electromagnetic pulse has been generated by the emitter. In order to prevent clutter from hiding the signal of interest, typical acoustic delay lines are designed with echoes in the 1 to $3 \mu$ s range, or assuming an acoustic velocity of $3000 \mathrm{~m} / \mathrm{s}$, a two way trip acoustic path of $9 \mathrm{~mm}$ (hence a $4.5 \mathrm{~mm}$ long acoustic path) at most. Such delays, which would be associated with $255 \mathrm{~m}$ deep interface in ice (assuming an electromagnetic velocity in ice of $170 \mathrm{~m} / \mu \mathrm{s}$ ), is only observed in cold regions such as an arctic environment [11]. All measurements were performed with a Malå ProEx GPR unit fitted with unshielded antennas, controlled by custom software. No hardware modification was needed on the GPR units, all measurement extraction is performed as digital signal post-processing on the recorded traces (Fig. 1).

However, rather than require a poor GPR setting with a very long measurement time window associated with a low sampling rate (considering that commercial GPR only allow for a few thousand samples to be recorded after each pulse is emitted), we have written dedicated opensource software - available at http://sourceforge.net/ projects/proexgprcontrol/ - by reverse engineering the

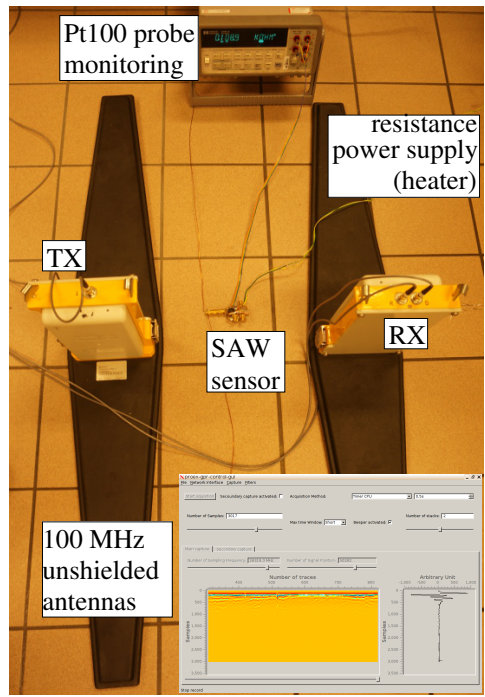

Fig. 1. Experimental setup: the SAW sensor is located between the emitter (TX) and the receiver (RX) of the unshielded GPR antennas. Locating the sensor on the surface yields poor coupling with the radiofrequency wave propagating preferentially in the higher permittivity concrete, yet provides a usable signal. Bottom right inset: screenshot of the graphical user interface of the custom proexgprcontrol software used for data acquisition. The command line interface is best suited for long term, autonomous data acquisition on embedded boards.

communication protocol, allowing for the definition of two distinct time windows, one for observing subsurface structures and the other one focusing on the delay line response. Both time windows use the full number of samples allowed by the commercial instrument, with the high associated sampling rate, and only the time offset is tuned for the record to start either before the pulse is generated by the emitter, or more than one microsecond after the pulse is generated to monitor the sensor response.

\section{MEASUREMENT RESUlTS}

Using SAW filters for purposes other than their original design means selecting a few random samples and trying the GPR interrogation process on each device. The selecting criteria is the central operating of the filter and the bandwidth of the filter which must match the associated characteristics of the signal emitted by the GPR. Three promising filters were selected from the TDK/Epcos list of components: both B3607 with a central frequency of $140 \mathrm{MHz}$ and a bandwidth of $6 \mathrm{MHz}$ and B5232 with a central frequency of $111 \mathrm{MHz}$ and a bandwidth of $1.15 \mathrm{MHz}$ for measurements with unshielded $100 \mathrm{MHz}$ antennas, and the B5087 with a central frequency of $192 \mathrm{MHz}$ but a bandwidth of 
$65 \mathrm{MHz}$. A GPR emitted signal is usually centered close to the mentioned operating frequency of a given dipole antenna set, with a usable bandwidth of about $10 \mathrm{MHz}$. Hence, the first filter sound the least appropriate in terms of operating frequency $-140 \mathrm{MHz}$ being between the two nominal frequencies of available antenna sets of 100 and $200 \mathrm{MHz}$, but the bandwidth of $6 \mathrm{MHz}$ or about $150 \mathrm{~ns}$ is well suited to the emitted signal. On the other hand, the second and third filter exhibits central frequencies close to the emitted pulse main frequency component, but the former filter is too narrow (a $1.15 \mathrm{MHz}$ wide filter requires a $870 \mathrm{~ns}$ long pulse to be efficiently loaded) and the latter is too wide (the inverse of $65 \mathrm{MHz}$ is a $15 \mathrm{~ns}$ time constant) to be efficiently loaded by the GPR pulse. Hence, our approach has been to use the $140 \mathrm{MHz}$ filter, despite the poor spectral density of the emitted pulse spectrum at this frequency (Fig. 2).
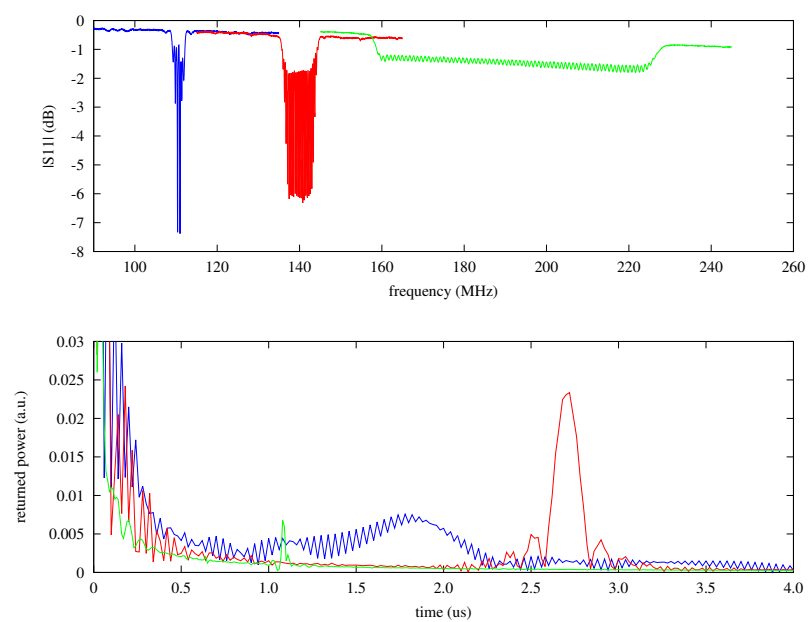

Fig. 2. $S_{11}$ reflected power from the filters (top), and returned echo spectra (bottom). The red and blue curves (bottom) respectively related to the B3607 and B5232 filters, were acquired with $100 \mathrm{MHz}$ unshielded antennas, the green curve related to the B5087 filter with $200 \mathrm{MHz}$ unshielded antennas.

Since SAW filters are not designed for a differential measurement approach with well defined multiple echoes, the demonstration proposed here will yield measurements results affected by the GPR to target distance: all experiments are performed with a static RADAR configuration. Dedicated reflective delay lines acting as cooperative targets would provide multiple echoes to get rid of the delay dependence with distance of the GPR emitter to SAW target in a differential measurement approach. Nevertheless, assuming a constant GPR to sensor distance, the single-echo SAW filter acts as a suitable cooperative target. The classical means of computing a time delay through cross correlation - practically implemented as the product of Fourier transforms of recorded signal segments for efficiency - is replaced with the computation of the phase of the Fourier transform at the main frequency component of the sensor response. Indeed, it is well known that the phase of the Fourier transform is representative in the time domain of the echo position. We hence compare the measurement of the sensor temperature using a reference Pt100 temperature probe with the unwrapped phase measurement of the Fourier transform of the part of the recorded signal including the echo generated by the SAW transducer acting as temperature sensor.
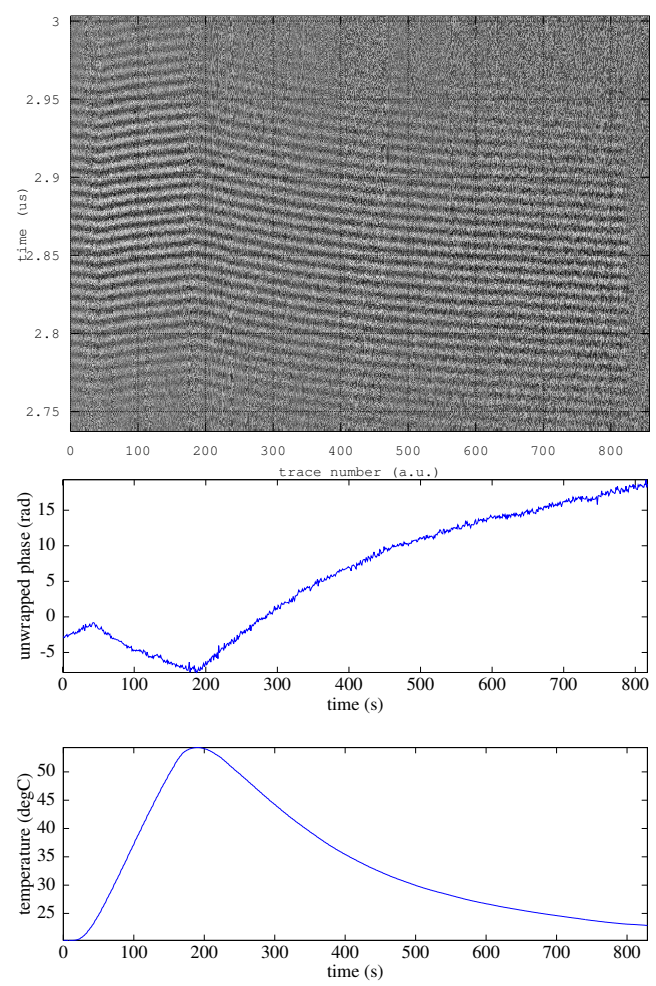

Fig. 3. Top: raw radargram, focusing on the region of interest including the SAW filter response as the temperature is first rising (traces 1 to 190) and then falling (traces 190 to the end). Bottom: phase of the Fourier component at the main signal returned by the SAW filter, and reference temperature measurement using a Pt100 probe glued on the SAW filter.

The echo position is representative of the physical quantity under investigation, in this particular case the temperature. Such investigations provide the basic for further developments including the investigating the acoustic wave velocity dependence with various physical parameters or impedance loading (Fig. 4). Indeed, transmission filters being 4-pole electrical components, the 
impedance load on the output port affects the reflective radiofrequency properties of the port the antenna is attached to. Using acoustic wave transducers as linear radiofrequency impedance converters have been investigated on dedicated coupled resonators [12] or delay lines [13] but never on general purpose, commercially available, acoustic filters. The charts of Fig. 4 focus on extreme cases of short, open and $50 \Omega$ loads in the context of remote switch detection, while a continuous capacitance load variation would allow for a continuous quantity monitoring (e.g. water level).
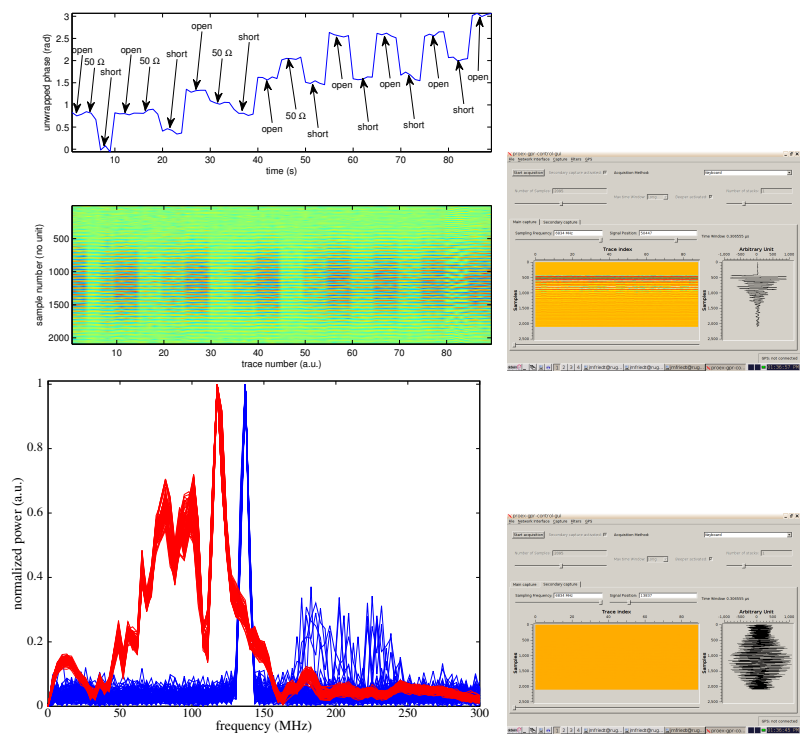

Fig. 4. Top left: phase of the echo as a function of impedance load on the second port of the filter. Open and short circuits induce significant phase shift and little amplitude variation, while a $50 \Omega$ load induces little phase shift with respect to the open circuit but a significant reflected echo amplitude drop due to the output port impedance matching. Right: two screenshots of the proexgprcontrol software graphical user interface with one (top) time window focusing on the emitted pulse and the second (bottom) time window focusing on the echo returned by the sensor. Bottom left: spectra of the emitted pulses (red) and of the returned echoes (blue), demonstrating the poor spectral overlap preventing a measurement at an extended range.

\section{CONCLUSION}

We have demonstrated the suitability of Surface Acoustic Wave (SAW) filters as introductory material for becoming familiar with such transducers acting as cooperative sensing targets to Ground Penetrating RADAR (GPR). For long term measurements using embedded electronics, a custom command line interface software has been implemented for controlling the Malå ProEx GPR unit in an autonomous mode. Although not specifically tuned for dedicated sensing purposes, SAW filters allow for testing signal processing techniques for complementing sub-surface interface investigation measurements with sensing capability requiring solely signal post-processing and no hardware update on commercial GPR units.

\section{ACKNOWLEDGMENT}

We acknowledge the TDK/Epcos company for kindly providing samples of the B39111B5232H310 and B39191B5087H810 filter references, and S. Ballandras (Frec'n'sys, Besançon, France) for fruitful discussions and improving the original manuscript. Acquisition of the Malå ProEx GPR unit was funded by a research grant from the Région de Franche-Comté

\section{REFERENCES}

[1] H. Stockman, "Communication by means of reflected power," Proceedings of the IRE, vol. 36, no. 10, pp. 1196-1204, 1948.

[2] C. T. Allen, K. Shi, and R. G. Plumb, "The use of groundpenetrating radar with a cooperative target," Geoscience and Remote Sensing, IEEE Transactions on, vol. 36, no. 5, pp. 1821$1825,1998$.

[3] G. Bruckner, A. Stelzer, L. Maurer, J. Biniasch, L. Reindl, and et al., "A high-temperature stable SAW identification tag for a pressure sensor and a low-cost interrogation unit," in 11th International Sensor Congress (SENSOR), Nuremberg, Germany, 2003, pp. 467-472.

[4] A. Pohl, R. Steindl, and L. Reindl, "The "intelligent tire" utilizing passive SAW sensors - mesurement of tire friction," IEEE Transactions on Instrumentation and Measurement, vol. 48, no. 6, pp. 1041-1046, December 1999.

[5] J. Beckley, V. Kalinin, M. Lee, and K. Volyansky, "Non-contact torque sensors based on SAW resonators," in IEEE International Frequency Control Symposium, New Orleans, USA, 2002, pp. 202-213.

[6] Y. Dong, W. Cheng, S. Wang, Y. Li, and G. Feng, "A multiresolution passive SAW chemical sensor," Sensors and Actuators $B$, vol. 76, pp. 130-133, 2001.

[7] J.-M. Friedt, A. Saintenoy, S. Chrétien, T. Baron, E. Lebrasseur, T. Laroche, S. Ballandras, and M. Griselin, "High-overtone bulk acoustic resonator as passive ground penetrating RADAR cooperative targets," J. Appl. Phys., vol. 113, no. 13, p. 134904, 2013.

[8] J.-M. Friedt, T. Rétornaz, S. Alzuaga, T. Baron, G. Martin, T. Laroche, S. Ballandras, M. Griselin, and J.-P. Simonnet, "Surface acoustic wave devices as passive buried sensors," Journal of Applied Physics, vol. 109, no. 3, p. 034905, 2011, available at http://jmfriedt.free.fr.

[9] C. Campbell, Surface Acoustic Wave Devices for mobile and wireless communications. Academic Press, 1998.

[10] D. Morgan, Surface acoustic wave filters, 2nd Ed. Academic Press, 2007.

[11] A. Saintenoy, J.-M. Friedt, A. Booth, F. Tolle, E. Bernard, D. Laffly, C. Marlin, and M. Griselin, "Deriving ice thickness, glacier volume and bedrock morphology of the austre lovénbreen (svalbard) using ground-penetrating radar," Near Surface Geophysics, vol. 11, no. 2, pp. 253-261, 2013.

[12] T. Laroche, G. Martin, W. Daniau, S. Ballandras, J.-M. Friedt, and J.-F. Leguen, "A cuopled-mode filter structure for wireless transceiver-sensors using reactive loads," in IEEE. Int. Freq. Control Symposium (IFCS), Baltimore, USA, 2012.

[13] L. Reindl, C. Ruppel, A. Kirmayr, N. Stockhausen, M. Hilhorst, and J. Balendonck, "Radio-requestable passive SAW watercontent sensor," IEEE Trans. on microwave theory and techniques, vol. 49, no. 4, pp. 803-808, 2001. 